\title{
Maturation of distinct neural components of the cognitive control network support early development of inhibitory control
}

\author{
Philipp Berger ${ }^{1,2 *}$, Angela D. Friederici ${ }^{1} \&$ Charlotte Grosse Wiesmann ${ }^{2}$ \\ ${ }^{1}$ Department of Neuropsychology, Max Planck Institute for Human Cognitive and Brain Sciences, \\ Leipzig, Germany \\ ${ }^{2}$ Research Group Milestones of Early Cognitive Development, Max Planck Institute for Human \\ Cognitive and Brain Sciences, Leipzig, Germany
}

\section{*Correspondence to:}

Philipp Berger

Max Planck Institute for Human Cognitive and Brain Science

Department of Neuropsychology

Stephanstraße 1a, 04103 Leipzig, Germany

Email: berger@cbs.mpg.de 


\begin{abstract}
Crucial for goal-directed behavior is the capacity to suppress impulses and predominant behavioral responses, called inhibitory control (IC). This ability emerges in early childhood, and a distinction according to neutral ('cold') and emotional ('hot') contexts has been suggested. Here, we ask which maturational changes in the child's brain underlie the emergence of this critical ability. We relate behavioral changes in 3-and 4-year-olds' 'hot' and 'cold' IC to brain maturation, using a multimodal approach that combines cortical and subcortical grey matter structure with white matter connectivity. Our results show that the maturation of distinct parts of the cognitive control brain network support early development of the different IC domains: Whereas 'cold' IC is related to frontoparietal regions and the left thalamus, 'hot' IC is associated with the left supramarginal gyrus and right thalamus. This dissociation of brain networks involved in 'cold' and 'hot' IC is confirmed by independent patterns of thalamocortical connectivity, supporting that IC in neutral and emotional settings relies on independent processes.
\end{abstract}

Keywords: Inhibitory Control, Executive Function, Brain Maturation, Cognitive Control Network 


\section{Introduction}

Inhibitory control (IC) is a fundamental cognitive function enabling us to voluntarily suppress impulses and predominant behavioral responses that are incompatible with our goals and intentions (Hofmann et al., 2012; Rothbart \& Posner, 1985). This ability is central to our daily lives, as it allows us to flexibly adjust to changing environmental conditions, forming a key prerequisite for goal-directed and adaptive behavior. Evidence from over three decades of research suggests that IC undergoes rapid development in the preschool period, particularly between the ages of 3 and 4 years, when children start passing standard IC tasks (Petersen et al., 2016). These early indices of adult-like cognitive control show long-term stability over the lifespan (Zelazo \& Carlson, 2012) and are highly predictive of later academic success and social adjustment (Diamond, 2013; Kim et al., 2013; Moffitt et al., 2011). Furthermore, in early childhood, the emergence of increasingly efficient IC has been argued to form the basis of significant improvements observed in many domains of cognition in a similar age range, including language (Ibbotson \& Kearvell-White, 2015), reasoning (Handley et al., 2004), decision-making (Cassotti et al., 2016), and social cognition (Benson et al., 2013). The emergence of IC in early childhood thus constitutes a key mechanism in human cognitive development. This highlights the importance of understanding the neurobiological preconditions for the emergence of this ability in early preschool-age. In the present study, we set out to investigate the maturation of brain structure and networks in the critical age range between 3 and 4 years that support the emergence of IC.

In early childhood, the human cerebral cortex undergoes complex developmental changes as a result of the interaction of complementary microbiological processes including synaptic pruning and myelination (Raznahan et al., 2011; Walhovd et al., 2017). These maturational processes are reflected in changes of cortical and subcortical grey matter (GM), and in enhanced interconnectedness through white matter (WM) fibre connections. Changes in local 
size and shape of cortical GM, as delineated by cortical thickness and surface area (Fischl \& Dale, 2000), as well as an increase in subcortical volume have shown robust associations with cognitive development in childhood (Noble et al., 2015; Pangelinan et al., 2011; Reiss et al., 1996; Walhovd et al., 2017). Similarly, the maturation of long-range WM pathways, reflected in higher connectivity between cortical brain regions, have been found to be reliably associated with the development in various domains of cognition during childhood (Grosse Wiesmann, Schreiber, et al., 2017; Johansen-Berg, 2010; Skeide et al., 2016). Together, these findings suggest that a multimodal view of GM and WM structures is necessary for understanding brain maturation processes associated with cognitive development. This interrelation of GM and WM structure with cognitive function has only rarely been studied in early childhood (but see Cafiero et al., 2019), and, in particular, multimodal research on the relation to early IC development is lacking. In the present study, we investigate the role of brain maturation in the early development of inhibitory functioning, using a combination of structural (sMRI) and diffusion-weighted Magnetic Resonance Imaging (dMRI).

In behavioral studies on early IC development, a distinction has been suggested between inhibition in neutral and emotional task contexts, referred to as 'cold' and 'hot' IC (Montroy et al., 2019; Zelazo \& Carlson, 2012). Traditionally, our understanding of IC is rooted in neuropsychological and clinical observations, investigating inhibitory functioning under purely cognitive and motivationally neutral ('cold') task conditions. In the classical Go-NoGo task, for example, children are required to perform a simple motor response when a given stimulus is displayed and to withhold this action when another stimulus is shown. Another line of research, however, has focused on IC processes that operate in motivationally and emotionally significant situations, and have been referred to as 'hot' IC. A prominent example is the classic Delay of Gratification task, in which children are required to inhibit the predominant behavioral impulse to choose an immediate emotionally salient reward (e.g., one marshmallow) in favor of a delayed larger reward (Mischel et al., 1989). Although the 
distinction between 'cold' and 'hot' inhibition is widely used in the behavioral developmental literature, it is debated whether these components share a common basis or address distinct and independent cognitive processes. Behavioral studies have shown that both 'hot' and 'cold' IC emerge in parallel between the ages of 3 and 4 years (Hongwanishkul et al., 2005) and may interact as part of a more general adaptive function (Zelazo \& Carlson, 2012). On the other hand, however, there is evidence highlighting a behavioral dissociation of the two task types (Montroy et al., 2019; Willoughby et al., 2011). Against this background, we investigated the maturation of brain structure and networks that support success in 'hot' and 'cold' IC tasks in 3- to 4-year-old children. Our aim was to clarify the brain-maturational changes underlying the emergence of the different facets of IC as well as addressing the question of the relation of 'hot' and 'cold' IC abilities. We reasoned that, if both abilities draw on the same cognitive process, the maturation of similar brain regions should be relevant for their development. If the maturation of different brain regions, however, support success on these tasks during development, this would support different underlying cognitive processes. To date, only little is known about the neural maturation processes underlying the emergence of IC in early childhood. In particular, the early preschool period from around 3 years of age that marks a critical take-off in IC abilities has not been studied to date, given the difficulty to conduct high-resolution neuroimaging methods like magnetic resonance imaging (MRI) with this age group. Candidate brain structures whose maturation may be crucial for the onset of IC in early childhood can thus only be derived drawing on the literature on mature IC in adults and older children.

Theoretical models of mature cognitive control have proposed a close connection with the prefrontal cortex (PFC) (Dempster, 1992; Diamond, 2013), as first evidenced by patient and lesion studies (Barceló \& Knight, 2002; Stuss \& Levine, 2002). This view was extended by studies using functional MRI, showing that the PFC is an important 'hub' in a larger neural network known to support inhibitory functioning in adults. This neural network, typically 
referred to as the superordinate Cognitive Control Network (sCCN) (Niendam et al., 2012), includes cortical structures in prefrontal areas, such as the anterior cingulate cortex (ACC) and the dorsolateral prefrontal cortex (DLPFC), as well as regions in the superior and inferior parietal cortices. In addition, subcortical regions, including the ventral striatum and thalamus, and their connections to relevant cortical areas were shown to be substantially involved in mature IC (Halassa \& Kastner, 2017; Somerville \& Casey, 2010). Traditionally, adult and patient studies have primarily focused on 'cold' IC task settings (Casey et al., 2001; Halassa \& Kastner, 2017), and it is currently unclear whether a similar network is recruited for control processes in emotionally and motivationally charged 'hot' contexts. In particular, a direct comparison of the neural processes of 'hot' and 'cold' IC task contexts is lacking as this distinction primarily stems from the classic tasks used in the developmental literature. So far, in adults, 'hot' IC abilities have been attributed to the orbitofrontal cortex (OFC) and frontostriatal connections, which are known to be involved in the flexible reappraisal of the affective or motivational significance of stimuli (Achterberg et al., 2016; Casey et al., 2011; Rolls, 2004). Studies in middle childhood and adolescence suggest some continuity of the functional involvement of the sCNN and PFC in 'cold' IC tasks at least from late childhood into adolescence (McKenna et al., 2017; Ordaz et al., 2013; Crone \& Steinbeis, 2017; Durston et al., 2002; Fiske \& Holmboe, 2019; Kharitonova et al., 2013; Rubia et al., 2007, 2013; Sheridan et al., 2014; Werchan \& Amso, 2017). For younger children, there is first evidence from a functional Near-Infrared Spectroscopy (fNIRS) study for a similar frontoparietal network that may be functionally involved between the age of 4 and 6 years (Mehnert et al., 2013). With regard to 'hot' IC, there is evidence that the integrity of frontostriatal connections predicts changes in the ability to delay gratification from middle childhood to adulthood (Achterberg et al., 2016; Van Den Bos et al., 2015). Together, these findings in adults and older children suggest that structural maturation of the sCCN and connections to subcortical areas in the striatum and thalamus may be critical for the emergence of IC in early childhood. 
Besides clarifying the factors that underlie the emergence of IC in its critical onset age between 3 and 4 years, early childhood is of particular interest for studying the 'hot-cold' distinction of IC, which builds on classic tasks that have been developed for this age group. Building on findings of behavioral dissociation, but parallel developmental trajectories of the two IC domains, this period constitutes the ideal window for a direct comparison of the neural networks and preconditions supporting 'cold' and 'hot' IC.

To summarize, in the present study, we investigate the role of brain maturation in the early development of inhibitory functioning, using a combination of structural (sMRI) and diffusion-weighted MRI (dMRI). We relate behavioral changes in 'cold' and 'hot' IC to the maturation of the PFC, OFC, and more broadly the sCCN, by investigating cortical and subcortical structure in combination with WM connectivity in the critical age of 3- to 4 years when IC emerges.

Our results show distinct neural networks within the $\mathrm{SCCN}$ involved in the development of 'cold' versus 'hot' IC. The development of 'cold' IC was uniquely related to the maturation of prefrontal and inferior parietal brain regions, as well as with the maturation of the left thalamus. In contrast, 'hot' IC was associated with maturation indices in the supramarginal gyrus and right thalamus. This dissociation was further confirmed by distinct and independent patterns of frontoparietal and thalamocortical white matter connectivity related to the IC domains. Our findings reveal that the emergence of 'cold' and 'hot' IC abilities is related to the structural maturation of distinct parts of the cognitive control network, functionally involved in IC in adults. We conclude that independent subnetworks of the $\mathrm{CCN}$ are involved in IC components, supporting the view that independent processes underlie the development of 'hot' and 'cold' IC. 


\section{Results}

\section{Behavioral results}

A behavioral assessment of 'hot' and 'cold' inhibitory functioning and codeveloping cognitive functions was conducted in 60 children aged 3 to 4 years (Grosse Wiesmann, Friederici, et al., 2017), from which a subsample of 37 children with usable MRI data was analyzed with respect to cortical brain structure (for details, see Methods). The children performed two standard tasks for 'cold' and 'hot' inhibitory functioning - a Go-NoGo task and a Delay of Gratification task (details see Methods). Performance in the 'cold' IC task showed a significant correlation with age (Spearman's $\rho=.45, \mathrm{p}<.001$ ), with 4-year-olds $(\mathrm{M}=0.94$, s.d. $=.10)$ performing significantly better than 3-year-olds $(\mathrm{M}=0.74$, s.d. $=0.31$; Mann-Whitney U-test, $\mathrm{U}=202.50, \mathrm{p}<0.001)$. Similarly, performance in the 'hot' IC task showed a significant correlation with age (Spearman's $\rho=.38, \mathrm{p}=.003$ ), with 4-year-olds $(\mathrm{M}=246.13$ s, s.d.=94.62) performing significantly better than 3-year-olds $(\mathrm{M}=183.96 \mathrm{~s}$, s.d.=119.35; Mann-Whitney U-test, $\mathrm{U}=296.50, \mathrm{p}=0.039)$. No significant correlation between 'hot' and 'cold' IC performance was found (Spearman's $\rho=.17, p=.224$ ).

\section{Analysis of cortical brain structure}

To test whether IC performance was related to cortical brain structure, we reconstructed cortical surface area and thickness from high-resolution anatomical MRI in the same children that participated in the behavioral task battery.

\section{'Cold' IC and cortical brain structure (Go-NoGo task)}

On the whole brain, we observed a positive correlation of children's 'cold' IC performance with their cortical surface area in the left rostral frontal cortex (RFC, BA 10), inferior temporal gyrus, precentral gyrus, and right posterior cingulate cortex (Table 1). Given our apriori hypotheses based on the brain regions relevant for 'cold' IC in adults, we additionally computed small-volume corrections within the regions of the sCCN (as reported in Niendam et al., 2012) and the PFC. In addition to the effect in the left RFC, this showed a positive 
correlation of children's 'cold' IC performance with their cortical surface area in the right RFC, right inferior parietal lobe (IPL), and in the caudal ACC (Table 1, Fig.1A and 1B). All effects remained significant when controlling for chronological age and gender. There were no significant effects for cortical thickness.

\section{'Hot' IC and cortical brain structure (Delay of Gratification task)}

To identify cortical brain structure associated with 'hot' IC development, we computed the linear relation of children's performance in the delay of gratification task with cortical surface area and thickness on the whole cortical surface. No significant correlation was found with either surface area or cortical thickness on the whole brain level. In a next step, we then tested whether children's 'hot' IC performance was related to cortical structure in our a-priori defined regions-of-interest. Using small-volume correction within the regions of the sCCN, PFC and OFC, we found a significant positive relation of children's 'hot' IC performance with their cortical surface area in the left supramarginal gyrus (Table 1, Fig1B), and no significant relations in the PFC or OFC. Again, the effect remained significant when controlling for chronological age and gender.

\section{Dissociation of 'cold' and 'hot' IC}

To test for the independence of the effects observed for 'cold' and 'hot' IC, we additionally controlled for performance in the other task, respectively. This showed that the regions of significant relation with 'cold' IC performance in bilateral RFC and right IPL were independent of 'hot' IC performance. Similarly, the 'hot' IC effect observed in the supramarginal gyrus was independent of 'cold' IC performance. Only the effect in caudal ACC did not remain significant when controlling for 'hot' IC performance.

\section{Analysis of subcortical brain structure}

To test whether IC performance was related to subcortical brain structure, we performed nonparametric partial correlational analysis, relating IC performance with the reconstructed 
volume of the thalamus and striatal areas, while controlling for age, gender and estimated total intracranial volume (eTIV). We observed a positive correlation between children's 'cold' IC performance and the volume of the left thalamus (Spearman's $\rho=.42, p=.013$, Figure 1C), whereas 'hot' IC performance was significantly related to the volume of the right thalamus (Spearman's $\rho=37, p=.013$, Figure 1C). The effect of 'cold' IC in the left thalamus remained significant after controlling for 'hot' IC performance. No significant correlation was found with the volume of striatal regions for 'hot' and 'cold' IC.

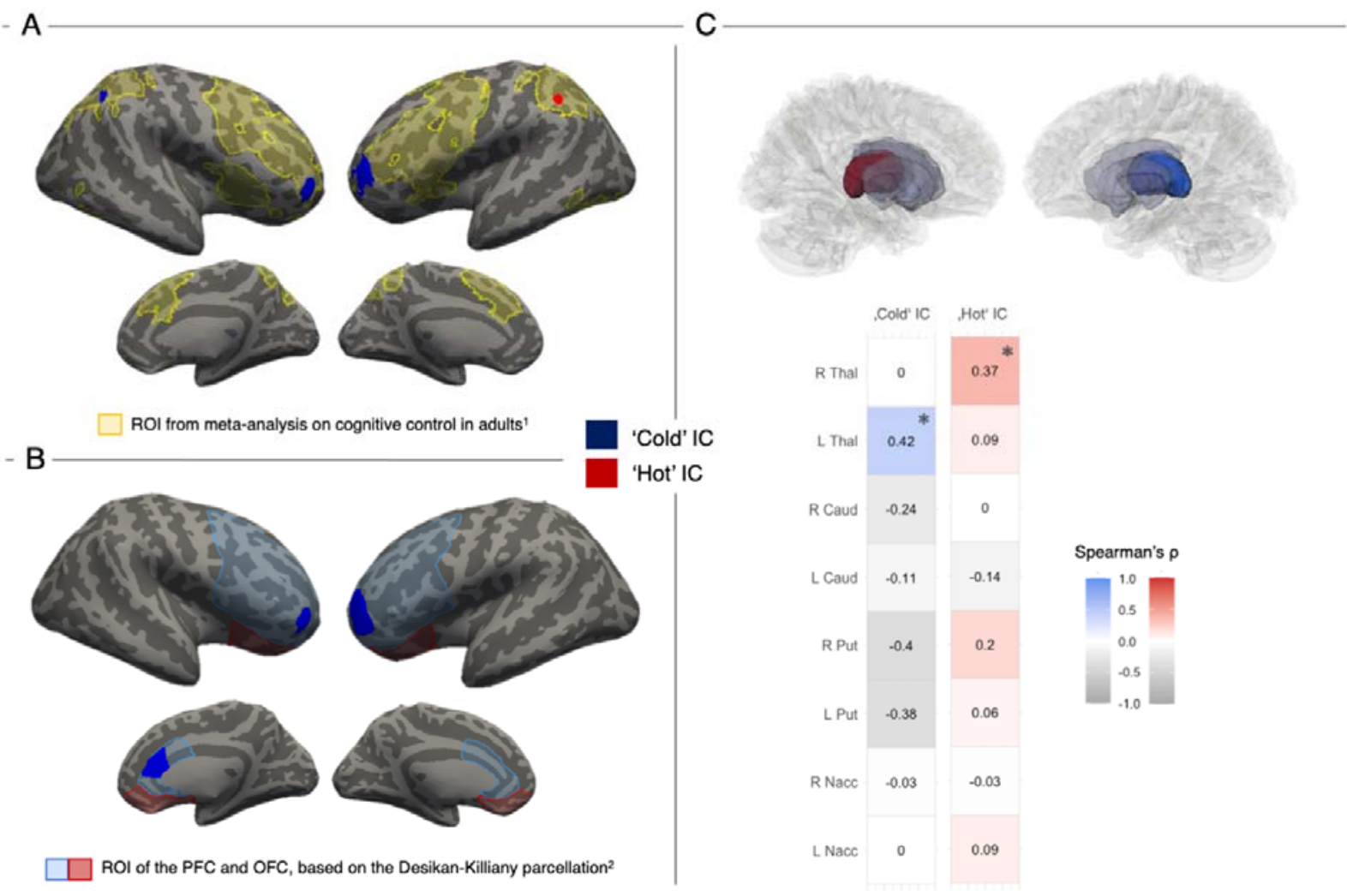

Figure 1. Relation of 'cold' (blue) and 'hot' (red) inhibitory control (IC) performance with cortical surface area and subcortical volume. (A) Small volume corrected linear relation of 'cold' IC performance (blue) with surface area in bilateral rostral frontal cortex (BA10) and right inferior parietal lobe and 'hot' IC performance (red) with cortical surface area in the left supramarginal gyrus within the regions of the superordinate Cognitive Control Network (sCCN; light yellow). (B) Small-volume corrected linear relation of 'cold' IC performance with surface area in the caudal ACC within the prefrontal cortex (PFC) mask (light blue) and medial orbitofrontal cortex (OFC) within the OFC mask (light red). All effects on the cortical surface are independent of children's age and 
gender, and cluster-size corrected at $\mathrm{P}<0.05$. (C) Results of non-parametric partial correlation analyses of 'cold' and 'hot' IC performance with volume of subcortical structures, color-coded for strength of positive association. A significant positive correlation of 'cold' IC (blue) with volume of the left thalamus, and 'hot' IC (red) with the right thalamus was revealed. These relations were independent of children's chronological age and gender. Abbreviations: Thal $=$ Thalamus, Caud $=$ Caudate Nucleus, Put $=$ Putamen, Nacc $=$ Nucleus accumbens.

\section{Analysis of white matter connectivity}

To investigate the structural connectivity of cortical and subcortical regions that were associated with 'hot' and 'cold' IC, respectively, we performed probabilistic tractography seeding in those regions. This analysis yielded a bilateral frontoparietal structural network connected to the cortical seed regions whose cortical structure had shown a linear relation to 'cold' and 'hot' IC performance (see Figure 2AB and Supplementary Material S1 for details). Through seeding in the thalamus, we identified thalamocortical structural connections in both hemispheres (Figure 2C).
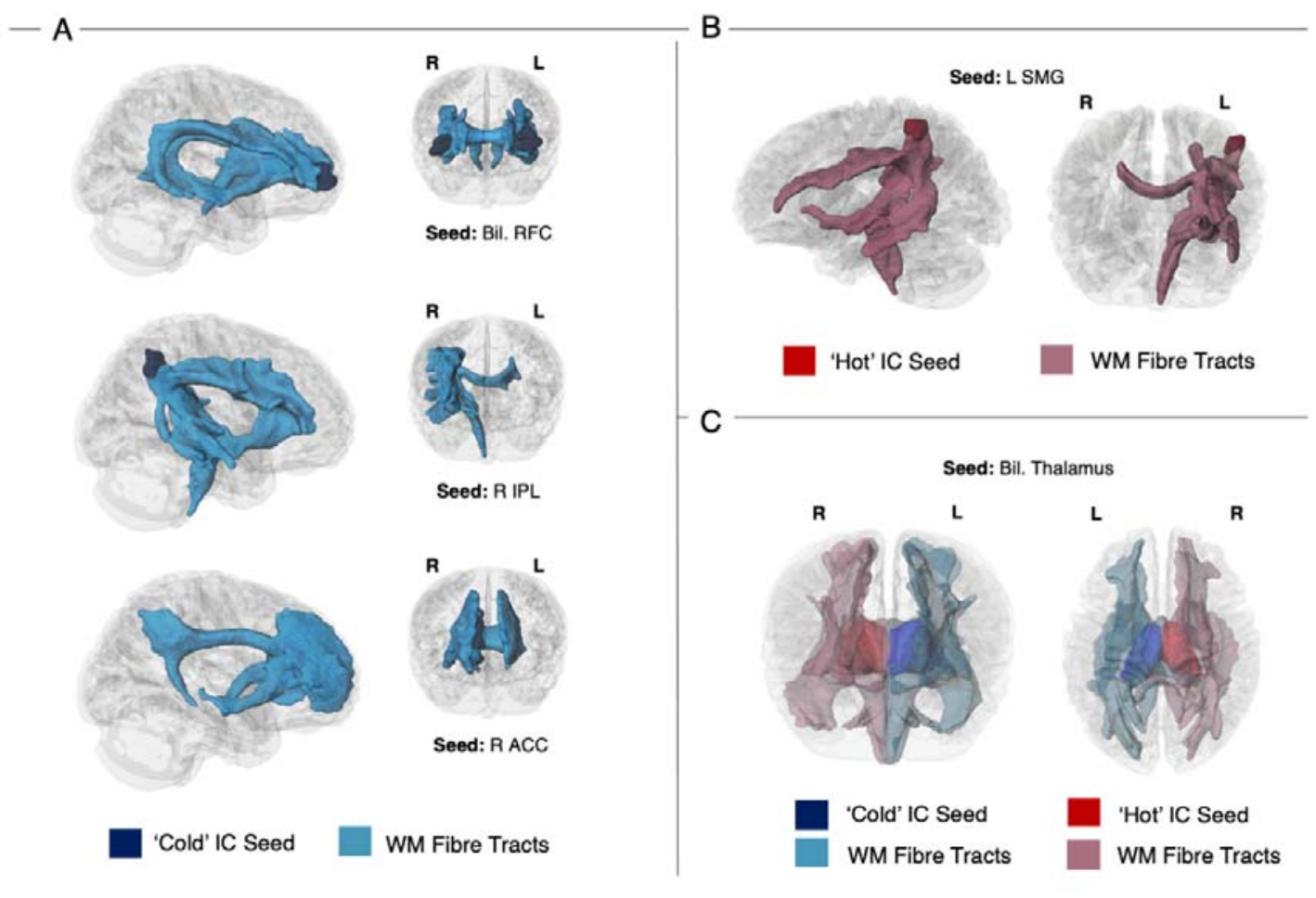

Figure 2. Streamline density maps resulting from probabilistic tractography seeded in brain regions showing a linear relation with 'cold' (blue) and 'hot' inhibitory control (IC) performance (red) on the cortical surface (A 
and $\mathrm{B}$, respectively) and subcortical volume (bilateral thalamus, C). A complete list of identified tracts can be found in the supplementary material. Abbreviations: $\mathrm{ACC}=$ Anterior Cingulate Cortex, IC = Inhibitory Control, $\mathrm{IPL}=$ Inferior Parietal Lobe, $\mathrm{OFC}=$ Orbitofrontal Cortex, $\mathrm{RFC}=$ Rostral Frontal Cortex, $\mathrm{SMG}=$ Supramarginal Gyrus, WM = White Matter.

Next, we wanted to specify how and where structural connectivity in these tracts was related to children's IC abilities. To this end, we correlated streamline densities obtained from tractography with the children's IC performance, while controlling for children's age, gender and surface area/volume of the seed region (see Methods for details). This showed a significant correlation of 'cold' IC performance with streamline density in the DLPFC indexing higher connectivity to this region from the seed in the IPL along the right superior longitudinal fasciculus (SLF) (see Figure 3B, Table 2). Furthermore, there was a significant correlation of 'cold' inhibitory functioning with streamline densities of the major forceps and left corticospinal tract, which connects the left thalamus with the motor cortex (see Figure 3A Table 2), independently of 'hot' IC performance. For 'hot' IC, we obtained a significant correlation with streamline density of the right superior and posterior thalamic radiation indexing higher connectivity from the seed in the right thalamus to the fronto-parietal and occipital cortex (see Figure 3C, Table 2).

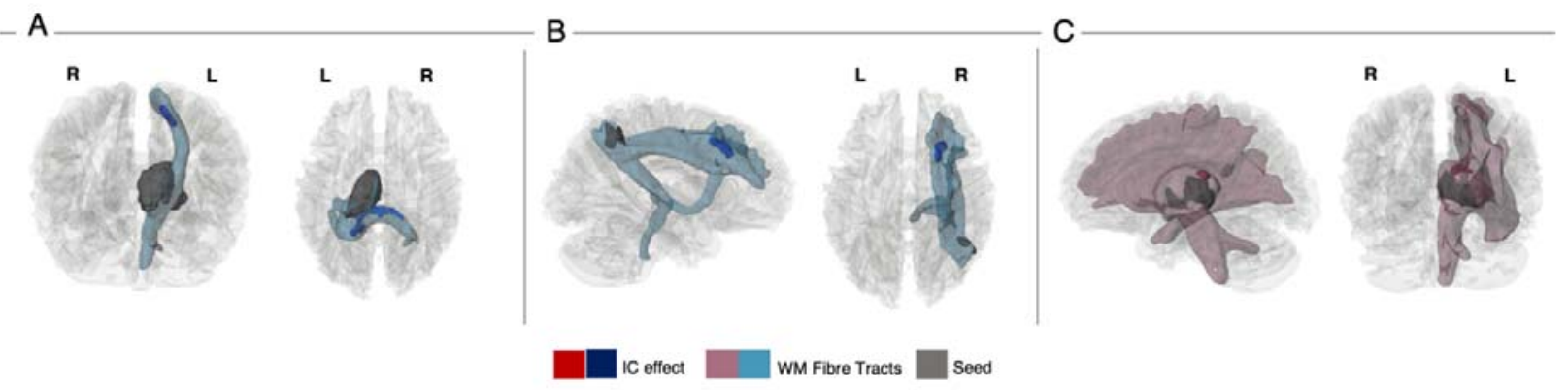

Figure 3. Correlation of streamline density with 'cold' (blue) and 'hot' inhibitory control (IC) performance (red). Results show a significant correlation of 'cold' IC with streamline density in the left corticospinal tract and forceps major (A), and in the right SLF (B). 'Hot' IC performance was correlated with streamline density of the right superior thalamic radiation (C). For visualization, white matter (WM) visitation maps (light red, light blue) are restricted to streamlines that pass through the significant cluster. The effects were independent of children's age and gender, as well as surface area/volume of the seed region. 


\section{Discussion}

IC constitutes a key prerequisite for humans to engage in goal-directed and adaptive behavior. A milestone of this ability is reached between the ages of 3 and 4 years, when children begin to master IC tasks both in neutral ('cold') and emotional ('hot') contexts. What are the neural maturation processes underlying the emergence of IC abilities in this critical age? Does the development of 'hot' and 'cold' IC share a common neural maturational basis, or do they build on distinct and independent processes that develop in parallel? To address these questions, we studied structural brain markers of GM and WM maturation in relation to 'hot' and 'cold' IC tasks at the critical age of 3 to 4 years. We found that the behavioral breakthrough in 'hot' and 'cold' IC was associated with the maturation of independent components of the cognitive control network, previously identified in adults (Niendam et al., 2012) and older children (McKenna et al., 2017). 'Cold' IC was related to maturation of the PFC, parietal lobe, and left thalamus, as well as frontoparietal and thalamocortical connections. 'Hot' IC, in contrast, was related to maturation of the supramarginal gyrus, right thalamus, and distinct thalamocortical connections. These findings shed light on the maturational basis of the emergence of IC, a central capacity for adaptive human behavior. Moreover, the independent brain networks involved in the emergence of 'hot' and 'cold' IC tasks support the view that IC relies on different processes for neutral and emotional settings. The emergence of 'cold' IC in early childhood was associated with the structural maturation of core regions in the sCCN. More specifically, an increase of surface area in prefrontal (i.e., ACC, bilateral RFC) and parietal cortices (i.e., right inferior parietal lobe), as well as an increase of volume in the left thalamus supported the marked developmental improvement observed on classic 'cold' IC tasks observed between 3 and 4 years. A similar brain network has previously been found to be functionally involved in mature 'cold' IC tasks in adults and late childhood (McKenna et al., 2017; Niendam et al., 2012). These findings support that the breakthrough observed in 'cold' IC measures in preschool children (Petersen et al., 2016), 
such as in variants of the Go-NoGo task, reflects a development towards mature cognitive control, and is based on neuromaturational changes of brain structure and connectivity in the preschool years. Changes in the structural connectivity of the above regions implicated in the early emergence of 'cold' IC revealed how the relevant regions are integrated in structural networks. This analysis yielded both frontoparietal and thalamocortical pathways, connecting important hubs of the sCCN. In particular, 'cold' IC performance was related to connectivity of the SLF connecting the DLPFC with the inferior parietal lobe, the posterior corpus callosum (i.e., major forceps) connecting the two hemispheres, and connections between the left thalamus and motor cortex through the left corticospinal tract. These results suggest that, in addition to cortical maturation in IC processing regions, the extent to which these regions are connected to each other and to the thalamus via association, projection and commissural fibres yields a mechanism for the emergence of mature IC. Our findings highlight the importance of white matter fiber connections that enable communication between core regions of cognitive control in prefrontal and parietal cortices and the thalamus. In particular, the SLF has been shown to provide the prefrontal cortex with information on visual space and visuo-motor function processed in the inferior parietal cortices, which may contribute to the regulation of spatial attention (Bisley \& Goldberg, 2003; Mesulam, 1981; Posner et al., 1984). The importance of the SLF for cognitive control is further supported by research on pathological conditions, showing that structural connectivity of the SLF is altered in disorders such as Obsessive-Compulsive Disorder (Gan et al., 2017) and Attention Deficit/Hyperactivity Disorder (ADHD; Gehricke et al., 2017; Hamilton et al., 2008). Specifically, research in ADHD has revealed that dysfunctional coupling in the SLF is associated with saccadic abnormalities (Fried et al., 2014; Matsuo et al., 2015) and increased response time variability (Wolfers et al., 2015). The corticospinal tract, in contrast, has functionally been linked to the control of voluntary movements (Kolb \& Whishaw, 2009), and alterations of the corticospinal tract have been associated with the hyperactivity syndrome in 
ADHD (Bu et al., 2020). Our results, therefore, suggest that brain structures subserving different functional components of cognitive control, including attention regulation and motor control, mature in early childhood that might enable young children to first master behavioral IC tasks in cognitive contexts.

In contrast to IC in neutral contexts, inhibition in an emotionally and motivationally highstake situation was related to the maturation of brain regions in the $\mathrm{sCCN}$ that were distinct from those observed for 'cold' IC. These included the supramarginal gyrus and right thalamus. This distinction was confirmed by a double dissociation in that the main reported effects were independent of performance in the respective other IC domain. This was also supported by a distinct structural network for 'hot' IC development, which was associated with the connectivity of the right thalamus with frontoparietal and occipital cortices via the superior and posterior thalamic radiation. Through ascending and descending tracts from the cerebral cortex the thalamic radiations integrate information throughout the brain, and are known to play a role in cognitive control and attention (Brandes-Aitken et al., 2019; Chaddock-Heyman et al., 2013; Stave et al., 2017).

In adults, the OFC and frontostriatal connections had previously been found to be functionally involved in IC in motivationally or emotionally-laden situations that require the inhibition of direct incentive needs to achieve a higher-value reward (Achterberg et al., 2016; Casey et al., 2011). In preschoolers, however, the maturation of these structures was not significantly associated with emerging 'hot' IC (see also Supplementary Material S2). Our data thus suggest that the developmental breakthrough in 'hot' IC observed in the critical age range between 3 and 4 years is mediated by the maturation of distinct components of the sCCN, rather than by a network specific for mature 'hot' IC. Indeed, besides the representation of incentive value emphasized in adults, 'hot' IC is thought to require the cognitive control of approach-avoidance tendencies (Zelazo \& Carlson, 2020). For example, to master the Delay of Gratification task, children must not only represent and navigate between the values of 
immediate and delayed rewards, but also be able to suppress their motor responses appropriately and, if necessary, reorient their attention in a different direction to achieve the goal. Our finding of early maturational processes of the supramarginal gyrus, a region implicated in attentional control (Corbetta et al., 2008), and cortical connections of the thalamus to parietal regions and the motor cortex suggests that controlling approachavoidance tendencies may be critical for the emergence of 'hot' IC in early childhood. Whether and from which age the maturation of frontostriatal connections (Achterberg et al., 2016; Casey et al., 2011) may be relevant to the early development of 'hot' IC abilities remains a question for future investigation.

In sum, the present findings support that independent parts of the cognitive control network, which mature around a similar age, support the emergence of 'cold' and 'hot' IC. This supports a dissociation of the processes underlying 'cold' and 'hot' IC, explaining low behavioral associations of IC in 'hot' and cold' task situations but synchronous development of the two capacities (Montroy et al., 2019; Willoughby et al., 2011; Zelazo \& Carlson, 2012). Moreover, this is in line with a dissociation of these abilities observed in clinical populations (Bechara, 2004; Eslinger et al., 2004). By providing a better understanding of the neural foundations for the emergence of inhibitory functioning and of its cognitive and neural architecture, our findings may foster a better understanding of developmental disorders with specific impairments in IC, such as ADHD, which has been found to differentially impair 'hot' and 'cold' IC performances (Antonini et al., 2015; Hobson et al., 2011).

\section{Methods}

\section{Participants}

MRI data and behavioral data of 37 typically developing 3-and 4-year-old children were analyzed for the present study (median $=4.06$ years, range: $3.07-4.58$ years; 20 female). The behavioral assessment was conducted in a total sample of $n=60$ children aged 3 and 4 
years(Grosse Wiesmann, Friederici, et al., 2017) from which we excluded children because they 1) did not participate in both IC tasks $(n=1), 2)$ did not participate in or aborted the MRI $(n=9), 3)$ showed incidental neurological findings $(n=1), 4)$ showed motion artifacts in the sMRI data $(\mathrm{n}=11)$ (Grosse Wiesmann et al., 2020) or 5) dMRI data, detected by visual inspection (Grosse Wiesmann, Schreiber, et al., 2017). One additional child was excluded due to an MRI acquisition error. Parental informed consent was obtained for all children, and the study was approved by the Ethics Committee at the Faculty of Medicine of the University of Leipzig.

\section{Assessment of IC}

The children performed two standard tests of 'cold' and 'hot' IC - a Go-NoGo task (Rakoczy, 2010) and a Delay of Gratification task(Mischel \& Ebbesen, 1970), which have been described in detail in previous studies (Grosse Wiesmann, Friederici, et al., 2017; Grosse Wiesmann, Schreiber, et al., 2017). Briefly, in the Go-NoGo task, children were asked to perform actions a duck puppet asked them to do (for example, 'Clap your hands!'), but not to do anything the nasty crocodile asked them to. A d-prime value was calculated with correct NoGo-trials as hits and incorrect Go-trials as false alarms $(\mathrm{M}=0.875$, s.d.=0.178). One child had to be excluded from further analyses because they did not provide complete data on the task. For the Delay of Gratification task, children asked to wait in front of highly desirable object (gummy bears or chocolate bars) for 5 minutes to receive a bigger reward. One child had to be excluded from further analyses because they did not provide complete data on the task. The children's mean waiting time was $M=226 \square$ s (s.d.=111s). To ensure comparability across task scores, we computed standardized scores of task performance in the Go-NoGo task and the Delay of Gratification task. Note that the data on these tasks were non-normally distributed because of the developmental breakthrough observed in the age range between 3 and 4 years. Therefore, we made sure that the statistical tests conducted were either nonparametric or that the data met the assumptions (i.e., normality of residuals). 


\section{MRI data acquisition}

MRI data were acquired on a 3-T Siemens scanner (Siemens MRT Trio series) using a 32channel head coil. The acquisition protocols are described in detail in previous studies (Grosse Wiesmann et al., 2020; Grosse Wiesmann, Schreiber, et al., 2017). High-resolution 3D T1-weighted MRI images were acquired using the MP2RAGE sequence (Marques et al., 2010) at $1.2 \times 1 \times 1 \mathrm{~mm}$ resolution (Grosse Wiesmann et al., 2020). Furthermore, dMRI data were acquired using the multiplexed echo planar imaging sequence (Feinberg et al., 2010) with a resolution of $1.9 \square \mathrm{mm}$ isotropic (Grosse Wiesmann, Schreiber, et al., 2017). A field map was acquired directly after the dMRI scan.

\section{SMRI data analysis}

\section{Cortical surface-based analyses}

To obtain measures of cortical thickness and surface area, we used the preprocessed brain images from a recent study using the current dataset (Grosse Wiesmann et al., 2020). In short, individual brain images were preprocessed in FreeSurfer 5.3.0 (http://surfer.nmr.mgh.harvard.edu) to reconstruct cortical surfaces and generate local estimates of cortical thickness and surface area following the standard surface-based stream (Fischl \& Dale, 2000). Surface area of the GM/WM boundary and cortical thickness, defined as the closest distance from the GM/WM boundary to the GM/CSF boundary, was calculated at each vertex. The resulting maps for cortical thickness and surface area were smoothed on the tessellated surfaces using a 10-mm FWHM Gaussian kernel. A common group template was created from the individual T1-weighted images of all children included in the analysis using ANTs (Avants et al., 2008). The individual cortical surfaces were registered to the common group template to allow for an accurate matching of local cortical thickness and surface area measures across participants.

\section{Subcortical volume analysis}


Individual brain images were processed in FreeSurfer 7.0 .0 (http://surfer.nmr.mgh.harvard.edu) to reconstruct subcortical volumes following the standard volume-based processing stream in FreeSurfer. The volume-based FreeSurfer processing stream conducts segmentation of several subcortical structures including the thalamus and striatum. Based on our hypotheses, we restricted subsequent analyses to the bilateral thalamus and structures of the striatum, including the bilateral putamen, bilateral caudate nucleus and bilateral nucleus accumbens. Quality control assessments of the thalamic and striatal reconstructions was conducted by two independent raters, yielding an interrater reliability of $\mathrm{M}_{\mathrm{ICC}}=.70$ (s.d.= .08 ). If the two raters agreed on a structure to show failures in segmentation this structure was excluded from the statistical analyses for the individual participant. This procedure resulted in the exclusion of the following number of participants per structure: $\mathrm{N}=1$ for the left caudate nucleus, $\mathrm{N}=2$ for the right caudate nucleus, $\mathrm{N}=2$ for the left putamen, $\mathrm{N}=2$ for the right putamen, $\mathrm{N}=5$ for the left nucleus accumbens, $\mathrm{N}=6$ for right nucleus accumbens, $\mathrm{N}=1$ for the left thalamus, and $\mathrm{N}=1$ for right thalamus.

\section{Statistical analysis}

The relation of cortical thickness and surface area, respectively, with our main variables ('cold' and 'hot' IC) were estimated in general linear models (GLM) using the tool mri_glmfit implemented in FreeSurfer. In the GLMs, we controlled for children's chronological age, and gender. Multiple comparison correction was applied with a clusterwise correction using the FreeSurfer tool mri_glmfit-sim, specifying a cluster-forming threshold of $\mathrm{P}<0.005$, clusterwise threshold of $\mathrm{P}<0.05$ (Greve \& Fischl, 2018), positive relation with surface area, bidirectional relation with cortical thickness, and additional correction for the analyses on two hemispheres (Grosse Wiesmann et al., 2020). For the clusterwise correction, a Monte Carlo simulation with 10,000 iterations was precomputed on the group template. Given our hypotheses for neural networks associated with 'cold' and 'hot' IC, respectively, we computed additional analyses using small-volume correction. In particular, we used a 
binarized map of the sCCN, derived from a meta-analysis on 'cold' inhibitory functioning in adults (Niendam et al., 2012). For this, we registered the original meta-analysis maps from the MNI space to our group template with the ANTs script WarpImageMultiTransform (http://stnava.github.io/ANTs/) and then projected them on the surface using the FreeSurfer tool mri_vol2surf (see Figure 1A). Furthermore, based on our specific hypotheses for PFC and OFC, we selected these as regions of interest based on the Desikan-Killiany parcellation (Desikan et al., 2006). The linear models for the relations of cortical thickness and surface area with 'cold' and 'hot' IC, respectively (as well as the analyses that included the covariates described above) were computed within the obtained masks with mri_glmfit as before. We checked for the normality of residuals using Kolmogorov-Smirnov tests.

The relation of subcortical volume of the thalamus and striatum with IC scores was assessed with non-parametric partial correlation analysis, using the 'ppcor' package (Kim, 2015) in R ( $\mathrm{R}$ Core Team, 2019). In the analysis, we used a one-sided (positive) test to indicate significant correlations, while controlling for children's chronological age, gender and eTIV, additionally correcting for the number of structures via false discovery rate (Benjamini \& Hochberg, 1995).

\section{dMRI data analysis}

\section{dMRI data processing}

To obtain measures of WM connectivity, we used the preprocessed dMRI data and analysis pipeline from another study using the current dataset (Grosse Wiesmann, Schreiber, et al., 2017). In short, after removing motion artefacts manually, motion itself was corrected for by rigidly aligning all volumes to the last one without diffusion weighting (b0) using flirt from the FSL software package (Jenkinson et al., 2002). Subsequently, the motion corrected dMRI data were rigidly aligned to the anatomical image, which again had been rigidly aligned to the Montreal Neurological Institute (MNI) standard space and was interpolated to $1 \square \mathrm{mm}$ isotropic voxel space. Distortions were corrected using the corresponding field map. 


\section{Seed region derivation}

To see within which tracts the significant clusters from the sMRI analyses were located, these regions were taken as seeds for probabilistic tractography. For this, we projected the significant regions derived in the cortical surface-based and volume-based analysis on the GM/WM boundary in the individual subject's brain using the FreeSurfer tool label2label. After transforming the label files to volume, we rigidly aligned them to the diffusion space using flirt from the FSL software package (Jenkinson et al., 2002).

\section{Statistical analysis}

Tractography was run with MRtrix (Tournier et al., 2012) using Constrained Spherical Deconvolution as a local model (Tournier et al., 2004) with the default parameters, as in Grosse Wiesmann et al. (2017). This procedure resulted in streamline density maps for each seed region and subject. To ensure that the identified correlations were not outlier-driven, we masked the streamline density maps of the individual subjects with a common group mask imposing that at least half the subjects have nonzero values in every voxel. The individual subjects' masked streamline density maps were then correlated with IC scores using GLMs with non-paremtric permutation tests in FSL randomise (Winkler et al., 2014), while controlling for the mean volume in the seed region of the tractography. This was done in order to ensure that correlations with streamline density were not driven by the correlation of the IC score and GM structure found in the sMRI analysis. In addition, we controlled for age and gender by including them as covariates in the linear model. Reported clusters in the tract volumes were significant at $\mathrm{P}<0.005$ at voxel-level and exceeded a cluster size significant at $\mathrm{P}<0.05$, in addition taking to account the number of streamline density maps according to Bonferroni correction. We localized and named the clusters and tracts based on the MRI Atlas of Human White Matter (Oishi et al., 2011). 


\section{References}

Achterberg, M., Peper, J. S., van Duijvenvoorde, A. C. K., Mandl, R. C. W., \& Crone, E. A. (2016). Frontostriatal white matter integrity predicts development of delay of gratification: A longitudinal study. Journal of Neuroscience, 36(6), 1954-1961. https://doi.org/10.1523/JNEUROSCI.3459-15.2016

Antonini, T. N., Becker, S. P., Tamm, L., \& Epstein, J. N. (2015). Hot and Cool Executive Functions in Children with Attention Deficit/Hyperactivity Disorder and Comorbid Oppositional Defiant Disorder. Journal of the International Neuropsychological Society $\square$ : JINS, 21(8), 584-595. https://doi.org/10.1017/S1355617715000752

Avants, B. B., Epstein, C. L., Grossman, M., \& Gee, J. C. (2008). Symmetric diffeomorphic image registration with cross-correlation: Evaluating automated labeling of elderly and neurodegenerative brain. Medical Image Analysis, 12(1), 26-41. https://doi.org/10.1016/j.media.2007.06.004

Barceló, F., \& Knight, R. T. (2002). Both random and perseverative errors underlie WCST deficits in prefrontal patients. Neuropsychologia, 40(3), 349-356. https://doi.org/10.1016/S0028-3932(01)00110-5

Bechara, A. (2004). The role of emotion in decision-making: Evidence from neurological patients with orbitofrontal damage. Brain and Cognition, 55(1), 30-40. https://doi.org/10.1016/j.bandc.2003.04.001

Benjamini, Y., \& Hochberg, Y. (1995). Controlling the False Discovery Rate: A Practical and Powerful Approach to Multiple Testing. Journal of the Royal Statistical Society: Series B (Methodological), 57(1), 289-300. https://doi.org/10.1111/j.2517-6161.1995.tb02031.x

Benson, J. E., Sabbagh, M. A., Carlson, S. M., \& Zelazo, P. D. (2013). Individual differences in executive functioning predict preschoolers' improvement from theory-of-mind training. Developmental Psychology, 49(9), 1615-1627. https://doi.org/10.1037/a0031056

Bisley, J. W., \& Goldberg, M. E. (2003). Neuronal activity in the lateral intraparietal area and spatial attention. Science, 299(5603), 81-86.

Brandes-Aitken, A., Anguera, J. A., Chang, Y.-S., Demopoulos, C., Owen, J. P., Gazzaley, A., Mukherjee, P., \& Marco, E. J. (2019). White matter microstructure associations of cognitive and visuomotor control in children: A sensory processing perspective. Frontiers in integrative neuroscience, 12, 65.

Bu, X., Yang, C., Liang, K., Lin, Q., Lu, L., Zhang, L., Li, H., Gao, Y., Tang, S., Hu, X., \& others. (2020). Quantitative tractography reveals changes in the corticospinal tract in drugnaive children with attention-deficit/hyperactivity disorder. Journal of psychiatry \& neuroscience: $J P N, 45(2), 134$.

Cafiero, R., Brauer, J., Anwander, A., \& Friederici, A. D. (2019). The concurrence of cortical surface area expansion and white matter myelination in human brain development. Cerebral Cortex, 29(2), 827-837.

Casey, B. J., Durston, S., \& Fossella, J. A. (2001). Evidence for a mechanistic model of cognitive control. Clinical Neuroscience Research, 1(4), 267-282. https://doi.org/10.1016/S1566-2772(01)00013-5

Casey, B. J., Somerville, L. H., Gotlib, I. H., Ayduk, O., Franklin, N. T., Askren, M. K., Jonides, J., Berman, M. G., Wilson, N. L., Teslovich, T., Glover, G., Zayas, V., Mischel, W., $\&$ Shoda, Y. (2011). Behavioral and neural correlates of delay of gratification 40 years later. Proceedings of the National Academy of Sciences of the United States of America, 108(36), 
14998-15003. https://doi.org/10.1073/pnas.1108561108

Casey, B. J., Trainor, R. J., Orendi, J. L., Schubert, A. B., Nystrom, L. E., Giedd, J. N., Castellanos, F. X., Haxby, J. V., Noll, D. C., Cohen, J. D., Forman, S. D., Dahl, R. E., \& Rapoport, J. L. (1997). A developmental functional MRI study of prefrontal activation during performance of a Go-No-Go task. Journal of Cognitive Neuroscience, 9(6), 835-847. https://doi.org/10.1162/jocn.1997.9.6.835

Cassotti, M., Agogué, M., Camarda, A., Houdé, O., \& Borst, G. (2016). Inhibitory Control as a Core Process of Creative Problem Solving and Idea Generation from Childhood to Adulthood. New Directions for Child and Adolescent Development, 2016(151), 61-72. https://doi.org/10.1002/cad.20153

Chaddock-Heyman, L., Erickson, K. I., Voss, M. W., Powers, J. P., Knecht, A. M., Hillman, C. H., \& Kramer, A. F. (2013). White matter microstructure is associated with cognitive control in children. Biological psychology, 94(1), 109-115. https://doi.org/10.1016/j.biopsycho.2013.05.008

Corbetta, M., Patel, G., \& Shulman, G. L. (2008). The Reorienting System of the Human Brain: From Environment to Theory of Mind. Neuron, 58(3), 306-324. https://doi.org/10.1016/j.neuron.2008.04.017

Crone, E. A., \& Steinbeis, N. (2017). Neural perspectives on cognitive control development during childhood and adolescence. Trends in cognitive sciences, 21(3), 205-215.

Dempster, F. N. (1992). The rise and fall of the inhibitory mechanism: Toward a unified theory of cognitive development and aging. Developmental Review, 12(1), 45-75. https://doi.org/10.1016/0273-2297(92)90003-K

Desikan, R. S., Ségonne, F., Fischl, B., Quinn, B. T., Dickerson, B. C., Blacker, D., Buckner, R. L., Dale, A. M., Maguire, R. P., Hyman, B. T., Albert, M. S., \& Killiany, R. J. (2006). An automated labeling system for subdividing the human cerebral cortex on MRI scans into gyral based regions of interest. NeuroImage, 31(3), 968-980. https://doi.org/10.1016/j.neuroimage.2006.01.021

Diamond, A. (2013). Executive Functions. Annual review of psychology, 64, 135. https://doi.org/10.1146/ANNUREV-PSYCH-113011-143750

Durston, S., Thomas, K. M., Yang, Y., Ulu $\square$, A. M., Zimmerman, R. D., \& Casey, B. J. (2002). A neural basis for the development of inhibitory control. Developmental Science, 5(4), F9-F16. https://doi.org/10.1111/1467-7687.00235

Eslinger, P. J., Flaherty-Craig, C. V., \& Benton, A. L. (2004). Developmental outcomes after early prefrontal cortex damage. Brain and Cognition, 55(1), 84-103. https://doi.org/10.1016/S0278-2626(03)00281-1

Feinberg, D. A., Moeller, S., Smith, S. M., Auerbach, E., Ramanna, S., Glasser, M. F., Miller, K. L., Ugurbil, K., \& Yacoub, E. (2010). Multiplexed echo planar imaging for sub-second whole brain fmri and fast diffusion imaging. PLoS ONE, 5(12), e15710. https://doi.org/10.1371/journal.pone.0015710

Fischl, B., \& Dale, A. M. (2000). Measuring the thickness of the human cerebral cortex from magnetic resonance images. Proceedings of the National Academy of Sciences of the United States of America, 97(20), 11050-11055. https://doi.org/10.1073/pnas.200033797

Fiske, A., \& Holmboe, K. (2019). Neural substrates of early executive function development. Developmental Review, 52, 42-62.

Fried, M., Tsitsiashvili, E., Bonneh, Y. S., Sterkin, A., Wygnanski-Jaffe, T., Epstein, T., \& 
Polat, U. (2014). ADHD subjects fail to suppress eye blinks and microsaccades while anticipating visual stimuli but recover with medication. Vision research, 101, 62-72.

Gan, J., Zhong, M., Fan, J., Liu, W., Niu, C., Cai, S., Zou, L., Wang, Y., Wang, Y., Tan, C., \& others. (2017). Abnormal white matter structural connectivity in adults with obsessivecompulsive disorder. Translational psychiatry, 7(3), e1062-e1062.

Gehricke, J.-G., Kruggel, F., Thampipop, T., Alejo, S. D., Tatos, E., Fallon, J., \& Muftuler, L. T. (2017). The brain anatomy of attention-deficit/hyperactivity disorder in young adults-a magnetic resonance imaging study. PloS one, 12(4), e0175433.

Greve, D. N., \& Fischl, B. (2018). False positive rates in surface-based anatomical analysis. NeuroImage, 171, 6-14. https://doi.org/10.1016/j.neuroimage.2017.12.072

Grosse Wiesmann, C., Friederici, A. D., Singer, T., \& Steinbeis, N. (2017). Implicit and explicit false belief development in preschool children. Developmental Science, 20(5), e12445. https://doi.org/10.1111/desc.12445

Grosse Wiesmann, C., Friederici, A. D., Singer, T., \& Steinbeis, N. (2020). Two systems for thinking about others' thoughts in the developing brain. Proceedings of the National Academy of Sciences of the United States of America, 117(12), 6928-6935. https://doi.org/10.1073/pnas.1916725117

Grosse Wiesmann, C., Schreiber, J., Singer, T., Steinbeis, N., \& Friederici, A. D. (2017). White matter maturation is associated with the emergence of Theory of Mind in early childhood. Nature Communications, 8(1), 1-10. https://doi.org/10.1038/ncomms14692

Halassa, M. M., \& Kastner, S. (2017a). Thalamic functions in distributed cognitive control. Nature Neuroscience, 20(12), 1669-1679. https://doi.org/10.1038/s41593-017-0020-1

Halassa, M. M., \& Kastner, S. (2017b). Thalamic functions in distributed cognitive control. Nature Neuroscience, 20(12), 1669-1679. https://doi.org/10.1038/s41593-017-0020-1

Hamilton, L. S., Levitt, J. G., O’Neill, J., Alger, J. R., Luders, E., Phillips, O. R., Caplan, R., Toga, A. W., McCracken, J., \& Narr, K. L. (2008). Reduced white matter integrity in attention-deficit hyperactivity disorder. Neuroreport, 19(17), 1705.

Handley, S. J., Capon, A., Beveridge, M., Dennis, I., \& Evans, J. S. B. (2004). Working memory, inhibitory control and the development of children's reasoning. Thinking \& Reasoning, 10(2), 175-195.

Hobson, C. W., Scott, S., \& Rubia, K. (2011). Investigation of cool and hot executive function in ODD/CD independently of ADHD. Journal of Child Psychology and Psychiatry, 52(10), 1035-1043. https://doi.org/10.1111/j.1469-7610.2011.02454.x

Hofmann, W., Schmeichel, B. J., \& Baddeley, A. D. (2012). Executive functions and selfregulation. Trends in Cognitive Sciences, 16(3), 174-180. https://doi.org/10.1016/j.tics.2012.01.006

Hongwanishkul, D., Happaney, K. R., Lee, W. S. C., \& Zelazo, P. D. (2005). Assessment of hot and cool executive function in young children: Age-related changes and individual differences. Developmental Neuropsychology, 28(2), 617-644. https://doi.org/10.1207/s15326942dn2802_4

Ibbotson, P., \& Kearvell-White, J. (2015). Inhibitory Control Predicts Grammatical Ability. PLOS ONE, 10(12), e0145030. https://doi.org/10.1371/journal.pone.0145030

Jenkinson, M., Bannister, P., Brady, M., \& Smith, S. (2002). Improved Optimization for the Robust and Accurate Linear Registration and Motion Correction of Brain Images. NeuroImage, 17(2), 825-841. https://doi.org/10.1006/nimg.2002.1132 
Johansen-Berg, H. (2010). Behavioural relevance of variation in white matter microstructure. Current Opinion in Neurology, 23(4), 351-358. https://doi.org/10.1097/WCO.0b013e32833b7631

Kharitonova, M., Martin, R. E., Gabrieli, J. D., \& Sheridan, M. A. (2013). Cortical graymatter thinning is associated with age-related improvements on executive function tasks. Developmental cognitive neuroscience, 6, 61-71.

Kim, S. (2015). ppcor: Partial and Semi-Partial (Part) Correlation. https://CRAN.Rproject.org/package $=$ ppcor

Kim, S., Nordling, J. K., Yoon, J. E., Boldt, L. J., \& Kochanska, G. (2013). Effortful control in ,hot" and „,cool“ tasks differentially predicts children's behavior problems and academic performance. Journal of Abnormal Child Psychology, 41(1), 43-56. https://doi.org/10.1007/s10802-012-9661-4

Kolb, B., \& Whishaw, I. Q. (2009). Fundamentals of human neuropsychology. Macmillan.

Luerssen, A., Gyurak, A., Ayduk, O., Wendelken, C., \& Bunge, S. A. (2015). Delay of gratification in childhood linked to cortical interactions with the nucleus accumbens. Social cognitive and affective neuroscience, 10(12), 1769-1776.

Marques, J. P., Kober, T., Krueger, G., van der Zwaag, W., Van de Moortele, P. F., \& Gruetter, R. (2010). MP2RAGE, a self bias-field corrected sequence for improved segmentation and T1-mapping at high field. NeuroImage, 49(2), 1271-1281. https://doi.org/10.1016/j.neuroimage.2009.10.002

Matsuo, Y., Watanabe, M., Taniike, M., Mohri, I., Kobashi, S., Tachibana, M., Kobayashi, Y., \& Kitamura, Y. (2015). Gap effect abnormalities during a visually guided pro-saccade task in children with attention deficit hyperactivity disorder. PloS one, 10(5), e0125573.

McKenna, R., Rushe, T., \& Woodcock, K. A. (2017). Informing the structure of executive function in children: A meta-analysis of functional neuroimaging data. Frontiers in Human Neuroscience, 11, 154. https://doi.org/10.3389/fnhum.2017.00154

Mehnert, J., Akhrif, A., Telkemeyer, S., Rossi, S., Schmitz, C. H., Steinbrink, J., Wartenburger, I., Obrig, H., \& Neufang, S. (2013). Developmental changes in brain activation and functional connectivity during response inhibition in the early childhood brain. Brain and Development, 35(10), 894-904.

Mesulam, M.-M. (1981). A cortical network for directed attention and unilateral neglect. Annals of Neurology: Official Journal of the American Neurological Association and the Child Neurology Society, 10(4), 309-325.

Mischel, W., \& Ebbesen, E. B. (1970). Attention in delay of gratification. Journal of Personality and Social Psychology, 16(2), 329-337. https://doi.org/10.1037/h0029815

Mischel, W., Shoda, Y., \& Rodriguez, M. L. (1989). Delay of gratification in children. Science, 244(4907), 933-938. https://doi.org/10.1126/science.2658056

Moffitt, T. E., Arseneault, L., Belsky, D., Dickson, N., Hancox, R. J., Harrington, H. L., Houts, R., Poulton, R., Roberts, B. W., Ross, S., Sears, M. R., Thomson, W. M., \& Caspi, A. (2011). A gradient of childhood self-control predicts health, wealth, and public safety. Proceedings of the National Academy of Sciences of the United States of America, 108(7), 2693-2698. https://doi.org/10.1073/pnas.1010076108

Montroy, J. J., Merz, E. C., Williams, J. M., Landry, S. H., Johnson, U. Y., Zucker, T. A., Assel, M., Taylor, H. B., Lonigan, C. J., Phillips, B. M., Clancy-Menchetti, J., Barnes, M. A., Eisenberg, N., Spinrad, T., Valiente, C., de Villiers, J., \& de Villiers, P. (2019). Hot and cool 
dimensionality of executive function: Model invariance across age and maternal education in preschool children. Early Childhood Research Quarterly, 49, 188-201. https://doi.org/10.1016/j.ecresq.2019.06.011

Niendam, T. A., Laird, A. R., Ray, K. L., Dean, Y. M., Glahn, D. C., \& Carter, C. S. (2012). Meta-analytic evidence for a superordinate cognitive control network subserving diverse executive functions. Cognitive, Affective and Behavioral Neuroscience, 12(2), 241-268. https://doi.org/10.3758/s13415-011-0083-5

Noble, K. G., Houston, S. M., Brito, N. H., Bartsch, H., Kan, E., Kuperman, J. M., Akshoomoff, N., Amaral, D. G., Bloss, C. S., Libiger, O., Schork, N. J., Murray, S. S., Casey, B. J., Chang, L., Ernst, T. M., Frazier, J. A., Gruen, J. R., Kennedy, D. N., Van Zijl, P., ... Sowell, E. R. (2015). Family income, parental education and brain structure in children and adolescents. Nature Neuroscience, 18(5), 773-778. https://doi.org/10.1038/nn.3983

Oishi, K., Faria, A., van Zijl, P. C. M., \& Mori, S. (2011). MRI Atlas of Human White Matter. Academic Press.

Olson, E. A., Collins, P. F., Hooper, C. J., Muetzel, R., Lim, K. O., \& Luciana, M. (2009). White matter integrity predicts delay discounting behavior in 9-to 23-year-olds: A diffusion tensor imaging study. Journal of cognitive neuroscience, 21(7), 1406-1421.

Ordaz, S. J., Foran, W., Velanova, K., \& Luna, B. (2013). Longitudinal growth curves of brain function underlying inhibitory control through adolescence. Journal of Neuroscience, 33(46), 18109-18124.

Pangelinan, M. M., Zhang, G., VanMeter, J. W., Clark, J. E., Hatfield, B. D., \& Haufler, A. J. (2011). Beyond age and gender: Relationships between cortical and subcortical brain volume and cognitive-motor abilities in school-age children. NeuroImage, 54(4), 3093-3100. https://doi.org/10.1016/j.neuroimage.2010.11.021

Petersen, I. T., Hoyniak, C. P., McQuillan, M. E., Bates, J. E., \& Staples, A. D. (2016). Measuring the development of inhibitory control: The challenge of heterotypic continuity. Developmental Review, 40, 25-71. https://doi.org/10.1016/j.dr.2016.02.001

Posner, M. I., Walker, J. A., Friedrich, F. J., \& Rafal, R. D. (1984). Effects of parietal injury on covert orienting of attention. Journal of neuroscience, 4(7), 1863-1874.

R Core Team. (2019). R: A Language and Environment for Statistical Computing. R Foundation for Statistical Computing. https://www.R-project.org/

Rakoczy, H. (2010). Executive function and the development of belief-desire psychology. Developmental Science, 13(4), 648-661. https://doi.org/10.1111/j.1467-7687.2009.00922.x

Raznahan, A., Lerch, J. P., Lee, N., Greenstein, D., Wallace, G. L., Stockman, M., Clasen, L., Shaw, P. W., \& Giedd, J. N. (2011). Patterns of coordinated anatomical change in human cortical development: A longitudinal neuroimaging study of maturational coupling. Neuron, 72(5), 873-884. https://doi.org/10.1016/j.neuron.2011.09.028

Reiss, A. L., Abrams, M. T., Singer, H. S., Ross, J. L., \& Denckla, M. B. (1996). Brain development, gender and IQ in children. Brain, 119(5), 1763-1774. https://doi.org/10.1093/brain/119.5.1763

Rolls, E. T. (2004). The functions of the orbitofrontal cortex. Brain and Cognition, 55(1), 1129. https://doi.org/10.1016/S0278-2626(03)00277-X

Rothbart, M. K., \& Posner, M. I. (1985). Temperament and the Development of SelfRegulation. In The Neuropsychology of Individual Differences (S. 93-123). Springer US. https://doi.org/10.1007/978-1-4899-3484-0_5 
Rubia, K., Lim, L., Ecker, C., Halari, R., Giampietro, V., Simmons, A., Brammer, M., \& Smith, A. (2013). Effects of age and gender on neural networks of motor response inhibition: From adolescence to mid-adulthood. NeuroImage, 83, 690-703. https://doi.org/10.1016/j.neuroimage.2013.06.078

Rubia, K., Smith, A. B., Taylor, E., \& Brammer, M. (2007). Linear age-correlated functional development of right inferior fronto-striato-cerebellar networks during response inhibition and anterior cingulate during error-related processes. Human brain mapping, 28(11), 1163-1177.

Sheridan, M., Kharitonova, M., Martin, R. E., Chatterjee, A., \& Gabrieli, J. D. (2014). Neural substrates of the development of cognitive control in children ages 5-10 years. Journal of Cognitive Neuroscience, 26(8), 1840-1850.

Skeide, M. A., Brauer, J., \& Friederici, A. D. (2016). Brain functional and structural predictors of language performance. Cerebral Cortex, 26(5), 2127-2139.

Somerville, L. H., \& Casey, B. J. (2010). Developmental neurobiology of cognitive control and motivational systems. Current Opinion in Neurobiology, 20(2), 236-241. https://doi.org/10.1016/j.conb.2010.01.006

Stave, E. A., De Bellis, M. D., Hooper, S. R., Woolley, D. P., Chang, S. K., \& Chen, S. D. (2017). Dimensions of attention associated with the microstructure of corona radiata white matter. Journal of child neurology, 32(5), 458-466.

Stuss, D. T., \& Levine, B. (2002). Adult clinical neuropsychology: Lessons from studies of the frontal lobes. Annual Review of Psychology, 53(1), 401-433. https://doi.org/10.1146/annurev.psych.53.100901.135220

Tournier, J. D., Calamante, F., \& Connelly, A. (2012). MRtrix: Diffusion tractography in crossing fiber regions. International Journal of Imaging Systems and Technology, 22(1), 5366. https://doi.org/10.1002/ima.22005

Tournier, J. D., Calamante, F., Gadian, D. G., \& Connelly, A. (2004). Direct estimation of the fiber orientation density function from diffusion-weighted MRI data using spherical deconvolution. NeuroImage, 23(3), 1176-1185. https://doi.org/10.1016/j.neuroimage.2004.07.037

Van Den Bos, W., Rodriguez, C. A., Schweitzer, J. B., \& McClure, S. M. (2015). Adolescent impatience decreases with increased frontostriatal connectivity. Proceedings of the National Academy of Sciences of the United States of America, 112(29), E3765-E3774. https://doi.org/10.1073/pnas.1423095112

Walhovd, K. B., Fjell, A. M., Giedd, J., Dale, A. M., \& Brown, T. T. (2017). Through Thick and Thin: A Need to Reconcile Contradictory Results on Trajectories in Human Cortical Development. Cerebral cortex (New York, N.Y. $\square:$ 1991), 27(2), 1472-1481. https://doi.org/10.1093/cercor/bhv301

Werchan, D. M., \& Amso, D. (2017). A novel ecological account of prefrontal cortex functional development. Psychological review, 124(6), 720.

Willoughby, M. T., Wirth, R. J., \& Blair, C. B. (2011). Contributions of modern measurement theory to measuring executive function in early childhood: An empirical demonstration. Journal of Experimental Child Psychology, 108(3), 414-435. https://doi.org/10.1016/j.jecp.2010.04.007

Winkler, A. M., Ridgway, G. R., Webster, M. A., Smith, S. M., \& Nichols, T. E. (2014). Permutation inference for the general linear model. NeuroImage, 92, 381-397. https://doi.org/10.1016/j.neuroimage.2014.01.060 
Wolfers, T., Onnink, A. M. H., Zwiers, M. P., Arias-Vasquez, A., Hoogman, M., Mostert, J. C., Kan, C. C., Slaats-Willemse, D., Buitelaar, J. K., \& Franke, B. (2015). Lower white matter microstructure in the superior longitudinal fasciculus is associated with increased response time variability in adults with attention-deficit/hyperactivity disorder. Journal of psychiatry \& neuroscience: $J P N, 40(5), 344$.

Zelazo, P. D., \& Carlson, S. M. (2012). Hot and Cool Executive Function in Childhood and Adolescence: Development and Plasticity. Child Development Perspectives, 6(4), 354-360. https://doi.org/10.1111/j.1750-8606.2012.00246.x

Zelazo, P. D., \& Carlson, S. M. (2020). The neurodevelopment of executive function skills: Implications for academic achievement gaps. Psychology \& Neuroscience, 13(3), 273-298. https://doi.org/10.1037/pne0000208 


\section{Tables}

Table 1. MNI coordinates, effect size, exact significance, and cluster size of significant relations between IC performance and cortical surface area.

\begin{tabular}{ccccc}
\hline Anatomical region & $\begin{array}{c}\text { Peak voxel coordinate } \\
\text { in MNI 305 space }(X, \\
\text { Y, Z) }\end{array}$ & $\begin{array}{c}\text { Correlation } \\
\text { in peak } \\
\text { voxel }\end{array}$ & $\begin{array}{c}\text { Cluster- } \\
\text { wise } \\
\text { P value }\end{array}$ & $\begin{array}{c}\text { Cluster-size } \\
\left(\text { in } \mathrm{mm}^{2}\right)\end{array}$ \\
\hline
\end{tabular}

Whole-brain analysis

'Cold' IC

$\begin{array}{lcccccc}\text { L RFC } & -35.4 & 57.1 & -6.2 & 0.431 & 0.00459 & 687.43 \\ \text { L inf. temporal lobe } & -63.3 & -43.2 & -20.2 & 0.544 & 0.00978 & 628.70 \\ \text { L precentral gyrus* } & -28.6 & -22.0 & 59.0 & 0.454 & 0.02247 & 550.10 \\ \text { R PCC* } & 13.6 & -23.9 & 35.9 & 0.480 & 0.00938 & 614.13\end{array}$

\section{Small-volume correction: $\mathrm{sCCN}$}

'Cold' IC

$\begin{array}{lrrrrrr}\text { L RFC* } & -33.1 & 56.2 & -2.1 & 0.341 & 0.00020 & 376.86 \\ \text { R RFC* } & 32.2 & 53.8 & -4.7 & 0.358 & 0.00060 & 209.65 \\ \text { R IPL* } & 44.0 & -61.2 & 45.2 & 0.390 & 0.01460 & 136.17\end{array}$

'Hot' IC
L SMG*
$\begin{array}{lll}-51.5 & -49.8 \quad 59.0\end{array}$
0.216
0.01720
113.21

Small-volume correction: PFC

'Cold' IC

$\begin{array}{lcccccc}\text { L RFC* } & -35.4 & 57.1 & -6.2 & 0.420 & 0.00010 & 687.43 \\ \text { R RFC* } & 38.0 & 58.8 & -7.1 & 0.387 & 0.03790 & 217.05 \\ \text { R caudal ACC } & 8.7 & 40.0 & 9.7 & 0.459 & 0.00120 & 374.78\end{array}$

Note. All effects are controlled for children's chronological age and gender. * Regions marked with an asterisk were independent from the other inhibitory control (IC) domain. Abbreviations: ACC = Anterior Cingulate Cortex, IPL = Inferior Parietal Lobe, OFC $=$ Orbitofrontal Cortex, PCC $=$ Posterior Cingulate Cortex, $\mathrm{RFC}=$ Rostral Frontal Cortex, SMG = Supramarginal Gyrus. 


\section{Acknowledgements}

We would like to thank Julia Werner, Anne Grigutsch, Lisa Uhlich, and Alina Kowald for their help with correcting segmentation and surface reconstruction in FreeSurfer, and Hung Nguyen Trong and Christiane Attig for their help with data acquisition and organization. 


\section{Author Contributions}

C.G.W. and A.D.F. conceived the research; C.G.W. designed the tasks and conducted the research; P.B. and C.G.W. analyzed the data; P.B. wrote the paper; and C.G.W. and A.D.F. edited the paper. 
bioRxiv preprint doi: https://doi.org/10.1101/2021.07.02.450852; this version posted July 26, 2021. The copyright holder for this preprint (which was not certified by peer review) is the author/funder. All rights reserved. No reuse allowed without permission.

\section{Competing Interests}

The authors declare no conflict of interest. 